University of Nebraska - Lincoln

DigitalCommons@University of Nebraska - Lincoln

Stephen Ducharme Publications

Research Papers in Physics and Astronomy

10-1996

\title{
Effect of beam attenuation on photorefractive grating erasure
}

\author{
Martin M Liphardt \\ University of Nebraska - Lincoln \\ Arosha Goonesekera \\ University of Nebraska-Lincoln \\ Stephen Ducharme \\ University of Nebraska-Lincoln, sducharme1@unl.edu \\ James M. Takacs \\ University of Nebraska-Lincoln, jtakacs1@unl.edu \\ Lei Zhang \\ University of Nebraska-Lincoln
}

Follow this and additional works at: https://digitalcommons.unl.edu/physicsducharme

Part of the Physics Commons

Liphardt, Martin M; Goonesekera, Arosha; Ducharme, Stephen; Takacs, James M.; and Zhang, Lei, "Effect of beam attenuation on photorefractive grating erasure" (1996). Stephen Ducharme Publications. 77. https://digitalcommons.unl.edu/physicsducharme/77

This Article is brought to you for free and open access by the Research Papers in Physics and Astronomy at DigitalCommons@University of Nebraska - Lincoln. It has been accepted for inclusion in Stephen Ducharme Publications by an authorized administrator of DigitalCommons@University of Nebraska - Lincoln. 


\title{
Effect of beam attenuation on photorefractive grating erasure
}

\author{
Martin Liphardt, Arosha Goonesekera, and Stephen Ducharme \\ Department of Physics and Astronomy, Center for Materials Research and Analysis, University of Nebraska-Lincoln, \\ Lincoln, Nebraska 68588-0111
}

James M. Takacs and Lei Zhang

Department of Chemistry, Center for Materials Research and Analysis, University of Nebraska-Lincoln, Lincoln, Nebraska 68588-0304

Received December 21, 1995; revised manuscript received March 28, 1996

\begin{abstract}
We investigate the influence of attenuation on the speed of erasure of photorefractive gratings by solving the coupled-wave equations in the undepleted pump approximation and by taking into account the attenuation and Gaussian intensity profile of all the beams. The extrinsic grating decay rate is significantly lower than the intrinsic photorefractive decay rate in samples with overall attenuation as low as $10 \%$. The Gaussian beam profiles of the readout and the erasing beams result in a further reduction of the extrinsic decay rate. The results of these calculations are used to determine the spectrum of intrinsic decay rates in a photorefractive polymer. (C) 1996 Optical Society of America.
\end{abstract}

\section{INTRODUCTION}

A great deal of theoretical and experimental research has been done to achieve a detailed understanding of the physical properties of photorefractive materials and to improve their performance. ${ }^{1,2}$ One important parameter is the speed at which a photorefractive grating can be erased (or written) because it reveals information about the dynamic processes involved in the effect, ${ }^{3,4}$ and it is also of practical interest because it limits the speed at which a photorefractive device may operate. Grating decay times are usually measured in four-wave mixing experiments in which two mutually coherent beams intersect to write a photorefractive grating, and a weak probe beam, incident at the Bragg diffraction angle, generates a diffracted signal beam, with an efficiency that depends on the grating strength. After the writing beams are turned off, the grating is erased with one of the writing beams, the probe beam, or another light beam. ${ }^{1}$ In this paper we derive an expression for the time dependence of the diffracted beam during erasure, using coupled-wave theory, ${ }^{5}$ and explicitly include the attenuation and the transverse Gaussian intensity profiles ${ }^{6}$ of all the beams. The effect of beam attenuation has been investigated by Baquedano et $a l .{ }^{7}$ for plane waves and unslanted dielectric gratings, and the effect of Gaussian beam profiles has been studied by Jaura et al..$^{8}$ for a simple geometry. Our analysis incorporates both effects in a general geometry, although some useful assumptions are retained, including neglect of the wave-front curvature of a Gaussian $\mathrm{TEM}_{00}$-mode beam. Appendix A explains the assumptions and their significance.

Basic photorefractive theory ${ }^{3,9}$ for a material with one kind of photorefractive trap predicts that the erasure of a sinusoidal plane grating is described by a single exponential of the form

$$
E_{\mathrm{SC}}(t)=E_{\mathrm{SC}}(0) \exp \left[-\Gamma_{i}(I) t\right],
$$

where the intrinsic speed of erasure $\Gamma_{i}(I)$ is given by

$$
\Gamma_{i}(I)=\frac{S_{\mathrm{ph}}}{\varepsilon \varepsilon_{0}} I W\left(\mathbf{k}, E_{a}\right)+\Gamma_{\text {dark }},
$$

where $E_{\mathrm{SC}}(t)$ is the amplitude of the space-charge field, $\Gamma_{\text {dark }}$ is the erasure rate due to dark conductivity, $S_{\mathrm{ph}}$ is the photosensitivity (the photoconductivity divided by the intensity $I$ of the erasing beam; $S_{\mathrm{ph}}$ can depend on intensity $\left.{ }^{10,11}\right), \varepsilon$ is the relative dielectric constant, $\varepsilon_{0}$ is the vacuum permittivity, and $W\left(\mathbf{k}, E_{a}\right)$ is a function that depends on the grating wave vector $\mathbf{k}$ and the externally applied electric field $E_{a} \cdot 1,3,4$

The speed of erasure depends on light intensity $I$, which is of special interest in this paper because attenuation (due to absorption or scattering) decreases the intensity of an erasing beam as the beam passes through the sample. Therefore a photorefractive grating is erased at a slower rate at the back of the sample than at the front. This fact is illustrated in Fig. 1, where the grating amplitude at various times is plotted as a function of the position $\alpha z$ in a sample of thickness $L=1 / \alpha$, with $\alpha$ being the attenuation coefficient. There is an increased departure from a uniform grating erasure (indicated by the dashed lines) as time progresses because the grating erases more slowly at the back of the sample, where the erasure intensity is lower. Note that at a given time the grating amplitude does not depend linearly on $\alpha z$, so that the speed is not simply determined by use of the average light intensity. Furthermore, the grating profile (Fig. 1) changes with time, which means that the decay is not exponential. The latter feature prompted the analysis presented in this paper because nonexponential decay is frequently offered as evidence of the presence of shallow traps. ${ }^{12,13}$ 

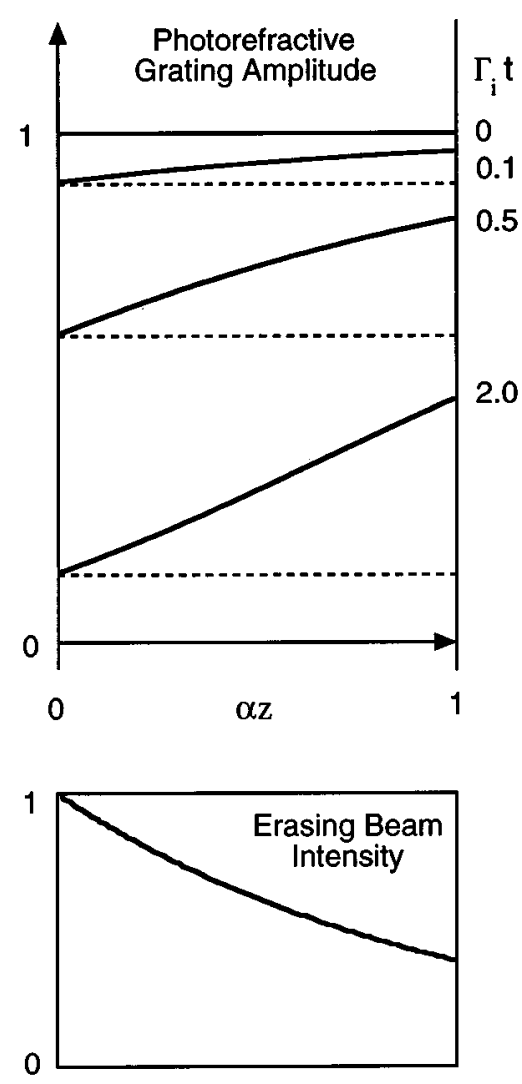

Fig. 1. Evolution of the normalized photorefractive grating amplitude during erasure with an attenuated erasing beam ( $\alpha L$ $=1$ ). A linear dependence of the intrinsic decay rate on light intensity is assumed ( $S_{p h}$ is a constant). The dashed lines indicate the evolution of the grating amplitude when attenuation is neglected.

\section{THEORY}

\section{A. One-Dimensional Coupled-Wave Equations (Plane} Waves)

To obtain the proper transient behavior of the diffracted beam, we modified the one-dimensional coupled-wave equations ${ }^{5}$ (in the slowly varying amplitude approximation) for explicit inclusion of a spatially varying coupling constant $\kappa(z, t)=a \delta n(z, t) \pi / \lambda$ :

$$
\begin{gathered}
\cos \left(\theta_{r}\right) \frac{\partial}{\partial z} R(z, t)+\frac{\alpha}{2} R(z, t)=-i \kappa(z, t) S(z, t) \\
R(0, t)=1, \\
\cos \left(\theta_{s}\right) \frac{\partial}{\partial z} S(z, t)+\frac{\alpha}{2} S(z, t)=-i \kappa(z, t) R(z, t) \\
S(0, t)=0,
\end{gathered}
$$

where $R(z, t)$ and $S(z, t)$ are the amplitudes of the probe and the diffracted beams, respectively; $\theta_{r}$ and $\theta_{s}$ are the respective internal angles that these beams make with the sample normal; $\alpha$ is the attenuation coefficient; $\delta n(z, t)$ is the amplitude of the index grating (see Fig. 2); and $a=1$ for $s$-polarized readout or $\cos \left(\theta_{r}-\theta_{s}\right)$ for $p$-polarized readout. The approximations that were made in arriving at Eqs. (3) and (4) and subsequent cal- culations are summarized in Appendix A. In the limit of small diffraction efficiency (undepleted pump regime) the solutions are given by

$$
\begin{gathered}
R(z)=\exp \left(\frac{-\alpha z}{2 c_{r}}\right), \\
S(z, t)=\frac{-i}{c_{s}} \exp \left(\frac{-\alpha z}{2 c_{s}}\right) \int_{0}^{z} \kappa\left(z^{\prime}, t\right) \\
\\
\quad \times \exp \left[\frac{\alpha z^{\prime}}{2}\left(\frac{1}{c_{s}}-\frac{1}{c_{r}}\right)\right] \mathrm{d} z^{\prime},
\end{gathered}
$$

where $c_{r}=\cos \left(\theta_{r}\right)$ and $c_{s}=\cos \left(\theta_{s}\right)$. In the case of a single photorefractive trap, we expect the intrinsic decay of the grating to be exponential at a rate $\Gamma_{i}(I)$ from Eq. (2) that depends linearly on light intensity:

$$
\kappa\left(z^{\prime}, t\right)=\kappa_{0} \exp \left\{-\Gamma_{i}\left[I\left(z^{\prime}\right)\right] t\right\}
$$

where $\kappa_{0}$ is the initial coupling strength, $I\left(z^{\prime}\right)$ $=I(0) \exp \left[-\alpha\left(L-z^{\prime}\right) / c_{e}\right]$ is the intensity of the erasing beam, $c_{e}=\cos \left(\theta_{e}\right)$, and $I(0)$ is the intensity of the erasing beam where it enters the sample $(z=L)$. Note that, for practical purposes, the erasing beam enters the sample from the side opposite the probe beam and that the probe beam is too weak to cause erasure. The integral in Eq. (6) may be computed after insertion of Eq. (7). This results in a nonexponential time dependence of the signal intensity $I_{D}(t)=S(L, t) S^{*}(L, t)$, the quantity measured in a four-wave mixing experiment. When we define the (time-dependent) apparent erasure rate $\Gamma_{a}(t)$ as

$$
\Gamma_{a}(t)=-\frac{1}{2} \frac{\mathrm{d}}{\mathrm{d} t} \ln \left[\frac{I_{D}<(t)}{I_{D}(0)}\right],
$$

nonexponential decay appears as the time dependence of $\Gamma_{a}(t) ; \Gamma_{a}(t)$ is a constant if the extrinsic decay is expo-

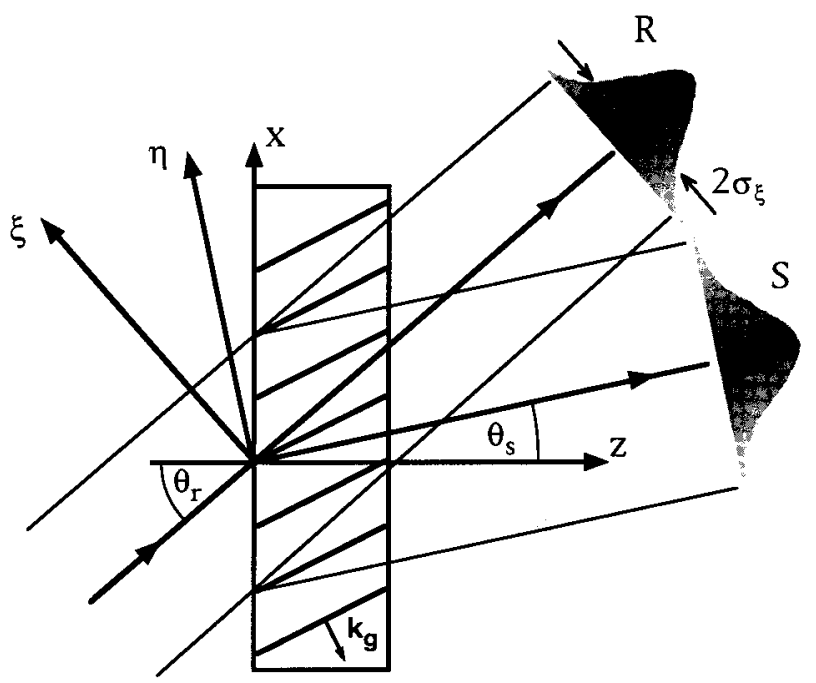

Fig. 2. Schematic drawing of a slanted grating with wave vector $k_{g}$ showing the probe beam $(R)$ and the diffracted beam $(S)$. The new coordinates $(\eta, \xi)$ are perpendicular to the beams $(R, S)$, respectively. 


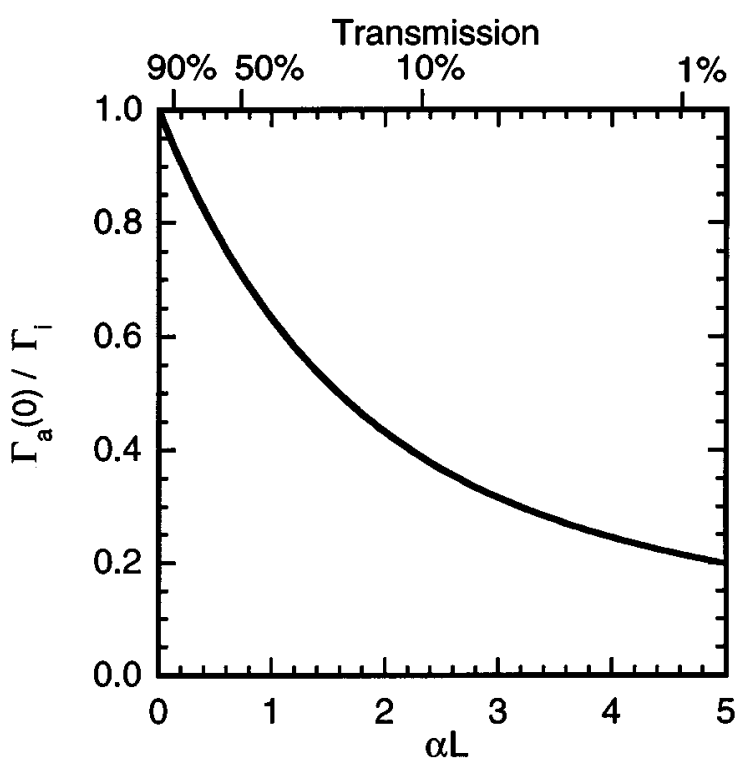

Fig. 3. Ratio of apparent decay rate to the intrinsic decay rate [Eq. (9)] as a function of $\alpha L$, the product of the attenuation coefficient and the sample length. The calculation includes an unslanted grating and plane-wave erasure, with neglect of the dark decay rate. The figure also applies to beams with a Gaussian profile, except for a constant factor that depends on the relative width of the reading and the erasing beams [Eq. (19)]

nential. Equation (8) can be used to find an analytic expression relating the intrinsic speed $\Gamma_{i}$ to the apparent speed at $t=0$ :

$$
\begin{aligned}
\Gamma_{a}(0)= & {\left[\frac{\Gamma_{i}^{\mathrm{ph}}}{\left.1-\frac{2 c_{s} c_{r}}{c_{e}\left(c_{s}-c_{r}\right)}\right]}\right.} \\
& \times\left\{\frac{1-\exp \left[\frac{-\alpha L}{2}\left(\frac{1}{c_{s}}-\frac{1}{c_{r}}+\frac{2}{c_{e}}\right)\right]}{1-\exp \left[\frac{-\alpha L}{2}\left(\frac{1}{c_{s}}-\frac{1}{c_{r}}\right)\right]}\right\} \\
& +\Gamma_{\text {dark }} .
\end{aligned}
$$

The intrinsic decay rate due to photoconduction $\Gamma_{i}{ }^{\mathrm{ph}}$, which is the quantity we want to measure, may be computed with this equation provided that the dark erasure rate $\Gamma_{\text {dark }}$, the attenuation coefficient $\alpha$, and all the beam angles are measured separately. The dependence of $\Gamma_{a}(0)$ on $\alpha L$ is shown in Fig. 3 for an unslanted grating, with neglect of the dark erasure rate $\Gamma_{\text {dark }}$. For samples with large attenuation the apparent speed is much lower than the intrinsic speed, but even a photorefractive sample with $90 \%$ bulk transmission exhibits a $6 \%$ reduction in apparent speed.

\section{B. Quasi-Three-Dimensional Coupled-Wave Equation (Gaussian Beams)}

To account for the effect of Gaussian beam profiles on grating erasure, the one-dimensional coupled-wave theory must be expanded to three dimensions ${ }^{6,14}$ with a coupling constant that depends on all three space coordinates $[\kappa=\kappa(x, y, z, t)]$. The following analysis is lim- ited to the case of weakly focused Gaussian beams with focal depth much greater than the sample thickness, so that one may approximate the Gaussian beam as a plane wave with nonuniform intensity distribution, neglecting the wave-front curvature (see Appendix A). In the slowly varying amplitude approximation the coupled-wave equations are

$$
\begin{aligned}
\sin \left(\theta_{r}\right) & \frac{\partial}{\partial x} R(x, y, z, t)+\cos \left(\theta_{r}\right) \frac{\partial}{\partial z} R(x, y, z, t) \\
+\frac{\alpha}{2} R(x, y, z, t) & =-i \kappa(x, y, z, t) S(x, y, z, t),
\end{aligned}
$$

$$
\begin{aligned}
\sin \left(\theta_{s}\right) & \frac{\partial}{\partial x} S(x, y, z, t)+\cos \left(\theta_{s}\right) \frac{\partial}{\partial z} S(x, y, z, t) \\
+ & \frac{\alpha}{2} S(x, y, z, t)=-i \kappa(x, y, z, t) R(x, y, z, t),
\end{aligned}
$$

with boundary conditions (at $z=0$; see Fig. 2):

$$
R(x, y, 0, t)=\exp \left(-\frac{x^{2}}{\sigma_{x}^{2}}-\frac{y^{2}}{\sigma_{y}^{2}}\right), \quad S(x, y, 0, t)=0
$$

where $\sigma_{x}=\sigma_{r} / \cos \left(\theta_{r}{ }^{\text {out }}\right)$ is the projection of the external $1 / e^{2}$ radius $\left(\sigma_{r}\right)$ of the reference-beam intensity onto the $x$ axis, where $\theta_{r}{ }^{\text {out }}$ is the external angle that the reference beam makes with the sample normal and $\sigma_{y}=\sigma_{r}$; in general, the beam profile is elliptical, with $\sigma_{x}>\sigma_{y}$, because of refraction at the $z=0$ interface. In the limit of small diffraction efficiencies (undepleted pump approximation) and by introduction of the new coordinates $\eta$ $=x \cos \left(\theta_{s}\right)-z \sin \left(\theta_{s}\right)$ and $\xi=x \cos \left(\theta_{r}\right)-z \sin \left(\theta_{r}\right)$ (see Fig. 2) the wave equations reduce to

$$
\frac{\partial}{\partial \eta} P(\xi, y, \eta)=-\frac{\alpha^{\prime}}{2} P(\xi, y, \eta),
$$

$$
\begin{aligned}
\frac{\partial}{\partial \xi} Q(\xi, y, \eta, t)= & +\frac{\alpha^{\prime}}{2} Q(\xi, y, \eta, t) \\
& +i \kappa^{\prime}(\xi, y, \eta, t) P(\xi, y, \eta),
\end{aligned}
$$

with $\alpha^{\prime}=\alpha /\left[\sin \left(\theta_{r}-\theta_{s}\right)\right], \kappa^{\prime}(\xi, y, \eta, t)=\kappa(\xi, y, \eta, t) /$ $\left[\sin \left(\theta_{r}-\theta_{s}\right)\right], P(\xi, y, \eta, \tau)=R(x, y, z, t)$, and $Q(\xi, y$, $\eta, t)=S(x, y, z, t)$. Note that the new coordinates $(\xi, \eta)$ are not orthogonal to each other; $\xi$ is perpendicular to the direction of propagation of the probe beam $R$, and $\eta$ is perpendicular to the direction of propagation of the diffracted beam $S$.

These equations can formally be solved to yield

$$
P(\xi, y, \eta)=\exp \left[-\frac{\xi^{2}}{\sigma_{\xi}^{2}}-\frac{y^{2}}{\sigma_{r}^{2}}-\frac{\alpha^{\prime}}{2}\left(\eta-\xi \frac{c_{s}}{c_{r}}\right)\right],
$$




$$
\begin{aligned}
Q(\xi, y, \eta, t)= & i \exp \left(\frac{\alpha^{\prime}}{2} \xi\right) \int_{\eta \frac{c_{r}}{c_{s}}}^{\xi} \exp \left(-\frac{\alpha^{\prime}}{2} \xi^{\prime}\right) \\
& \times \kappa^{\prime}\left(\xi^{\prime}, y, \eta, t\right) P\left(\xi^{\prime}, y, \eta\right) \mathrm{d} \xi^{\prime},
\end{aligned}
$$

with $\kappa^{\prime}(\xi, y, \eta, t)=\kappa_{0} \exp \left\{-\Gamma_{i}[I(\xi, y, \eta)] t\right\}$, and $\sigma_{\xi}$ $=\sigma_{x} \cos \left(\theta_{r}\right)$ is the $1 / e^{2}$ radius of the reference beam inside the sample measured in the $\xi$ direction.

At the output surface ( $z=L$; see Fig. 2$)$ the two new coordinates are not independent, and we find that

$$
\eta c_{r}-\xi c_{s}=L \sin \left(\theta_{r}-\theta_{s}\right),
$$

which can be used to eliminate the $\xi$ dependence of the diffracted amplitude $Q(\xi, y, \eta, t)$ at the output surface. Provided that the area of the detector that measures the power of the diffracted beam is large enough to capture essentially the whole beam, the output signal $I_{D}(t)$ of the detector is given by

$$
I_{D}(t)=\int_{-\infty}^{\infty} \int_{-\infty}^{\infty} Q_{z=L}(y, \eta, t) Q_{z=L} *(y, \eta, t) \mathrm{d} y \mathrm{~d} \eta,
$$

where $Q_{z=L}(y, \eta, t)$ is the amplitude of the diffracted beam at the output surface where Eq. (17) holds. Keeping in mind the assumptions summarized in Appendix A, we find that Eq. (18) applies to a general writing and erasing geometry. In particular, the direction of the erasing beam is arbitrary, although for computation of the integrals in Eqs. (16) and (18) the intensity distribution of the erasing beam must be expressed in terms of the new coordinates $(\xi, y, \eta)$, and this expression, in general, yields very complex integrals. Analytic expressions of the apparent decay rate at $t=0$ may be derived from Eq. (18) only for special cases. For the case of counterpropagating or copropagating erase and probe beams $\left(c_{r}\right.$ $=c_{e}$ ) with Gaussian beam profiles, we find that

$\Gamma_{a}(0)$

$$
\begin{aligned}
= & {\left[\frac{\Gamma_{i}{ }^{\mathrm{ph}}}{\left.1 \pm \frac{2 c_{s}}{\left(c_{s}-c_{r}\right)}\right]}\right.} \\
& \times\left\{\frac{\exp \left(-\frac{\alpha L}{c_{r}} \frac{1 \pm 1}{2}\right)-\exp \left[-\frac{\alpha L}{2}\left(\frac{1}{c_{s}} \mp \frac{1}{c_{r}}\right)\right]}{1-\exp \left[-\frac{\alpha L}{2}\left(\frac{1}{c_{s}}-\frac{1}{c_{r}}\right)\right]}\right\} \\
& \times\left(\frac{\sigma_{e}{ }^{2}}{\sigma_{e}{ }^{2}+\sigma_{r}^{2}}\right)+\Gamma_{\text {dark }},
\end{aligned}
$$

where $\Gamma_{i}{ }^{\text {ph }}$ is the intrinsic decay rate that is due to photoconduction at the peak intensity of the erasing beam with a Gaussian profile, the upper sign describes erasure by the reading beam (or a beam copropagating with the reading beam), and the lower sign describes erasure by a beam counterpropagating with the reading beam. [See Appendix A for a discussion of the assumptions inherent in Eq. (19)]. As expected, in the limit of large erasing beam width $\left(\sigma_{e} \gg \sigma_{r}\right)$, Eq. (19) reduces to Eq. (9) when the latter is evaluated with $c_{r}=c_{e}$. The additional fac- tor containing $\sigma_{e}+\sigma_{r}$ is the ratio of the erasing beam area to the sum of the erasing and the probe-beam areas.

Alternatively, the erasing beam can enter the sample from the top or the side so that it travels (approximately) perpendicular to the probe beam. This arrangement can reduce the effect of beam attenuation on grating erasure if the probe-beam width is smaller than the attenuation length. One can easily modify the derivation of Eqs. (16) and (18) to accommodate for this geometry by merely expressing the erasing beam amplitude in the new coordinates $\xi, y, \eta$. Assuming a plane-wave erasing beam traveling in the $x$ direction (see Fig. 2) and a symmetric writing geometry $\left(c_{r}=c_{s}\right)$, the following expression for the apparent decay rate may be derived:

$$
\begin{aligned}
\Gamma_{a}(0)= & \Gamma_{i} \operatorname{ph}\left[\frac{ \pm \cos \left(\theta_{s}\right)}{\alpha L \sin \left(\theta_{s}\right)}\right]\left\{\exp \left[\frac{ \pm \alpha L \sin \left(\theta_{s}\right)}{\cos \left(\theta_{s}\right)}\right]-1\right\} \\
& \times \exp \left[\frac{-\alpha d}{2}+\frac{\alpha^{2}{\sigma_{r}}^{2}}{2 \cos ^{2}\left(\theta_{s}\right)}\right]
\end{aligned}
$$

where $d$ is the $x$ dimension of the sample, $\Gamma_{i}^{\text {ph }}$ is the intrinsic decay rate due to photoconduction at the light intensity of the erasing beam where it enters the sample, and the plus sign (minus sign) corresponds to an erasing beam traveling in the negative (positive) $x$ direction (probe-and signal-beam angles are measured counterclockwise from the $z$ axis).

\section{Photoconductivity}

Measurements of the sample conductance also underestimate the intrinsic photoconductivity that is due to attenuation of the illuminating beam (see bottom of Fig. 1). One measures the conductance by applying a voltage across the sample and measuring the change in current when the sample is illuminated. ${ }^{11}$ If the direction of propagation of the illumination is parallel to the applied field, the effective resistance of the sample is the sum of the series resistances (thin slabs) of the sample, where the resistance is lowest at the front and highest at the back. Nonuniform illumination of the sample with electrode area $A_{0}$ causes the resistance to vary across each slab, and one obtains the resistance of each slab by summing over the parallel conductances. Assuming that a light beam with a Gaussian intensity profile of $1 / e^{2}$ area $A_{G}=\pi \sigma_{r}{ }^{2}$ is used to illuminate the sample, that $A_{G}$ $\ll A_{0}$, and that $\sigma_{\mathrm{ph}} \propto I$, we find the following for the effective resistance $R$ of the sample:

$$
R=\frac{1}{A_{0}} \int_{0}^{L} \frac{\mathrm{d} z}{\sigma_{d}+\frac{A_{G}}{2 A_{0}} \sigma_{\mathrm{ph}} \exp (-\alpha z)},
$$

where $\sigma_{d}$ is the dark conductivity and $\sigma_{\mathrm{ph}}$ is the photoconductivity at the peak light intensity at the front of the sample. After performing the integration, one can solve for the photoconductivity: 


$$
\sigma_{\mathrm{ph}}=\frac{2 L}{A_{G} R_{d}}\left\{\frac{1-\exp \left[\left(1-\frac{R}{R_{d}}\right) \alpha L\right]}{\exp \left(-\frac{R}{R_{d}} \alpha L\right)-1}\right\},
$$

where $R_{d}$ is the sample resistance in the dark.

Once the five quantities in Eq. (22) are measured, the intrinsic photoconductivity can be computed. In the limit of small attenuation $(\alpha L \ll 1)$ this equation reduces to the usual result $\left(2 A_{0} / A_{G}\right)\left(\sigma-\sigma_{d}\right)$, where $\sigma$ is the effective sample conductivity during sample illumination. Note that the effective resistance $R$ approaches the dark resistance $R_{d}$ in the limit of large attenuation.

\section{EXPERIMENT}

Two different photorefractive materials were investigated by use of a standard degenerate four-wave mixing technique (Fig. 4), the photorefractive polymer Bisphenol A 4,4'-nitroaminostilbene (BisA-NAS) with 29 wt.\% benzaldehyde-diphenyl hydrazone $(\mathrm{DEH})^{11,15}$ and a crystal of $\mathrm{BaTiO}_{3}$ (purchased in 1991 from Sanders Associates, Nashua, N.H.). Polymer samples had a thickness of $145 \mu \mathrm{m}$, and the crystal dimensions were $4.0 \mathrm{~mm} \times 4.5$ $\mathrm{mm} \times 5.5 \mathrm{~mm}$ with the $c$ axes oriented along the shortest

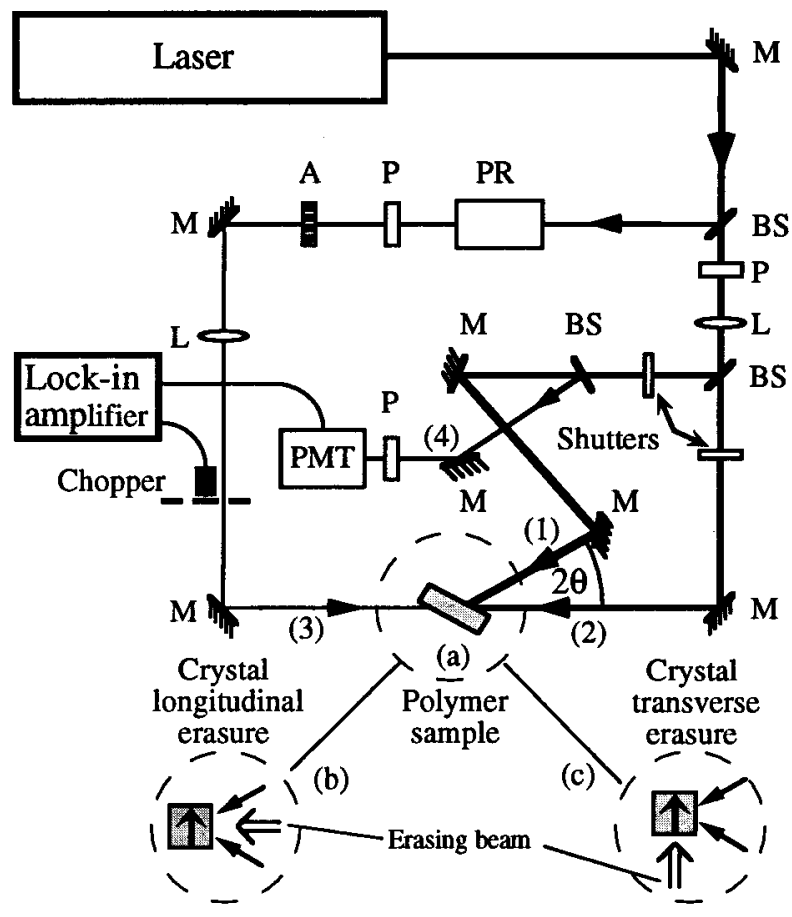

Fig. 4. Experimental setup of four-wave mixing measurements: L, lens; M, mirror; BS, beam splitter; P, polarizer; PR, polarization rotator; A, attenuator; PMT, photomultiplier tube. Beams (1) and (2) are the (s-polarized) writing beams, and beams (3) and (4) are the ( $p$-polarized) probe and diffracted beams, respectively. (a) Slanted geometry for polymer samples. An $\mathrm{Ar}^{+}$-laser-pumped dye laser was used for writing, reading, and erasing. (b), (c) Symmetric geometry of longitudinal and transverse erasure in $\mathrm{BaTiO}_{3}$ with crystal $c$ axis in the plane of writing beams and perpendicular to their bisector. A He-Ne laser was used for writing and reading, and the erasing beam was derived from an $\mathrm{Ar}^{+}$laser. dimension. Measurements with the polymer samples were performed with writing ( $s$-polarized light), reading ( $p$-polarized), and erasing ( $s$-polarized) beams from a cw dye laser (pumped by an $\mathrm{Ar}^{+}$laser) operating at a wavelength of $650 \mathrm{~nm}$ (other wavelengths were also used to obtain a spectrum). The polymer sample $(\alpha=204 \pm 8$ $\mathrm{cm}^{-1}$ ) was mounted in a slanted geometry ${ }^{11}$ with the erasing beam counterpropagating the read beam and with both beams having approximately equal width of $1.6 \mathrm{~mm}$ $\left(1 / e^{2}\right.$ diameter). The external angle of incidence was $40.0^{\circ}$ for one of the writing beams (beam 1 in Fig. 4) and $60.0^{\circ}$ for the reading, the writing, and the erasing beams (beams 2 and 3 in Fig. 4), respectively. The index of refraction was $1.73 \pm 0.04$ (determined by measurement of Brewster's angle), yielding internal angles of $21.3 \pm 0.5^{\circ}$ and $30.0 \pm 0.7^{\circ}$, respectively, and a grating period $\Lambda_{g}$ $=2.62 \pm 0.01 \mu \mathrm{m}$ at an angle of $64.1 \pm 0.6^{\circ}$ from the sample normal. The modulation depth $m=2 \sqrt{I_{1} I_{2}} /\left(I_{1}\right.$ $+I_{2}$ ) of the interference pattern of the writing beams was approximately 0.18 , and a dc voltage of $5.0 \mathrm{kV}$ was applied across the sample to align the nonlinear optical molecules and to provide drift-assisted photorefractive charge transport. ${ }^{16}$

For the $\mathrm{BaTiO}_{3}$ crystal a $\mathrm{He}-\mathrm{Ne}$ laser $(632.8 \mathrm{~nm})$ was used for writing ( $s$ polarized) and reading ( $p$ polarized), whereas erasing ( $s$ polarized) was done with the 514.5-nm line of an $\mathrm{Ar}^{+}$laser. No voltage was applied to the $\mathrm{BaTiO}_{3}$ crystal, and the attenuation coefficients were measured to be $\alpha=1.46 \pm 0.08 \mathrm{~cm}^{-1}$ at $632.8 \mathrm{~nm}$ and $\alpha=3.7 \pm 0.1 \mathrm{~cm}^{-1}$ at $514.5 \mathrm{~nm}$. A symmetric writing beam geometry was used for the $\mathrm{BaTiO}_{3}$ crystal, with the crystal $c$ axis oriented parallel to the grating wave vector (Fig. 4). The external angle between the writing beams was $10.0^{\circ}$, and the sample thickness was the longest crystal dimension $(5.5 \mathrm{~mm})$. Beam widths were 0.94 and 1.7 $\mathrm{mm}\left(1 / e^{2}\right.$ diameter $)$ for the reading and the writing beams, respectively. The index of refraction at $632.8 \mathrm{~nm}$ was ${ }^{17} n_{a}=2.412$, yielding an internal angle between the writing beams of $2.15 \pm 0.05^{\circ}$ and a grating period $\Lambda_{g}$ $=3.64 \pm 0.01 \mu \mathrm{m}$. The modulation depth was $m$ $=0.20$. The greatly expanded erasing beam (diameter $\approx 10 \mathrm{~mm}$ ) was oriented either parallel to the bisector of the writing beams (longitudinal case) or parallel to the crystal $c$ axis (transverse case).

\section{RESULTS AND DISCUSSION}

A typical decay curve (normalized to 1 at $t=0$ ) obtained from the polymer sample displays evident nonexponential decay [Fig. 5(a)]. The apparent decay rate at $t=0$, $\Gamma_{a}(0)$, was determined from the data by examination of a plot of the time derivative of the natural logarithm of the data [see Fig. 5(b) and Eq. (9)], and Eq. (19) was used to calculate the intrinsic decay rate $\Gamma_{i}^{\mathrm{ph}}$, which is illustrated in Fig. 5(a). Once $\Gamma_{i}{ }^{\mathrm{ph}}$ is known, all parameters [except for the initial signal $\left.I_{D}(0)\right]$ of the integral in Eq. (18) are determined so that the integral may be computed (numerically) to plot the diffracted intensity (at all times), as is shown in Fig. 5(a). Note that this procedure does not constitute a fit in the usual sense. Rather, it was assumed that the calculated data will match the experimental data at all times, including $t=0$, where the apparent 


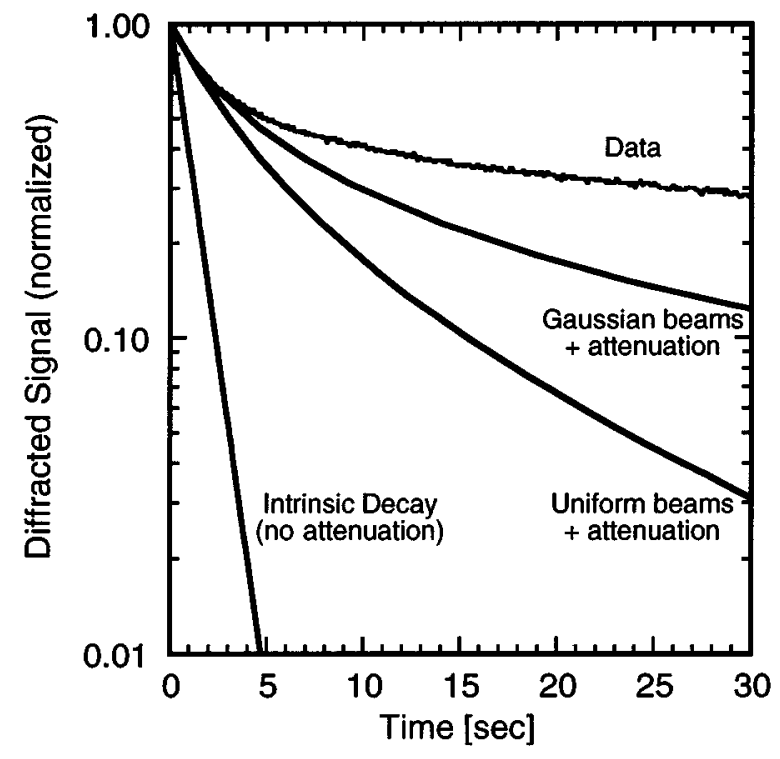

(a)

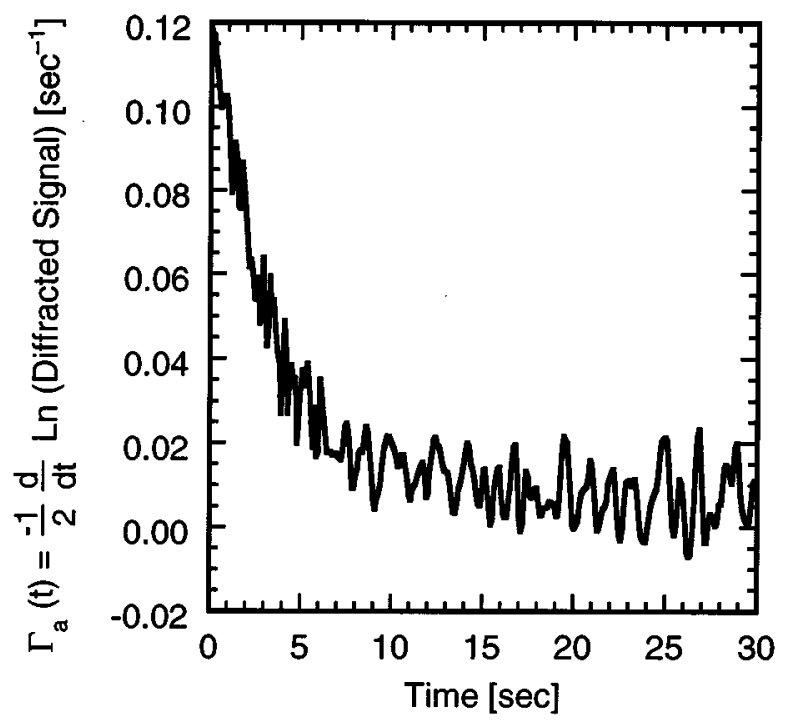

(b)

Fig. 5. (a) Experimental erasure data and calculated decay of the normalized intensity of the diffracted beam as a function of time. The sample was the polymer BisA-NAS:DEH and $\alpha L$ $=2.96$. The straight line is the intrinsic decay at a rate corresponding to the peak intensity $\left(0.18 \mathrm{~W} / \mathrm{cm}^{2}\right)$ of the erasing beam with a Gaussian profile at the input face. The two solid curves represent the calculated decay accounting for attenuation of plane-wave probe and erasing beams only (uniform beams +attenuation) and accounting for attenuation and beam profile of Gaussian probe and erasing beams (Gaussian beams +attenuation). (b) Apparent decay rate that is equal to the time derivative of the natural logarithm of experimental erasure data in (a).

decay rate was determined, and thus a good match is always achieved at early times. This procedure was chosen because only one parameter (besides the trivial initial signal strength), $\Gamma_{i}{ }^{\mathrm{ph}}$, needs to be determined to compute the integral in Eq. (18), and Eq. (19) makes it very convenient to obtain $\Gamma_{i}^{\mathrm{ph}}$ by inspection of the experimental data at $t=0$.
The calculated curve accounting for Gaussian beams and attenuation in Fig. 5(a) shows a qualitative improvement over a simple exponential, although it does not properly describe the data at all times. For the purpose of comparison we also plotted a theoretical curve, treating all the light beams as having a uniform intensity distribution [Eq. (6)], thus, singling out the effect of beam attenuation alone. Clearly, the Gaussian intensity distribution has a pronounced effect and needs to be included in the analysis. The solution [Eq. (18)] incorporating attenuation and Gaussian beam profiles did not match the data, which implies that the nonexponential decay is partially of intrinsic origin. This conclusion is supported by the temporal behavior of the grating decay in the dark (Fig. 6), which is clearly nonexponential. The intrinsic decay may be nonexponential because of, e.g., shallow traps, which also results in an intrinsic decay rate that is not linear in light intensity, ${ }^{12,13}$ as was indeed observed with this polymer. No attempt was made by us to use a modified time and light-intensity dependence of the intrinsic decay. We demonstrate here, however, that attenuation and beam profiles strongly affect erasure data, as is apparent by comparison of the calculated erasure curves with the assumed intrinsic exponential decay [Fig. 5(a)]. Therefore an analysis of erasure data that does not accurately account for attenuation and beam profiles cannot properly measure the intrinsic erasure rate or characterize intrinsic erasure.

Example decay curves for longitudinal and transverse erasure obtained from the $\mathrm{BaTiO}_{3}$ crystal are shown in Fig. 7, and the theoretical curves were computed from Eq. (19) in the limit of large erasing beam width for the longitudinal erasure and from Eq. (20) for the transverse erasure, as described above. The initial apparent speeds of both erasure schemes are approximately the same, but, as expected from the calculation [Eq. (18)], the two decay curves deviate from each other as time progresses be-

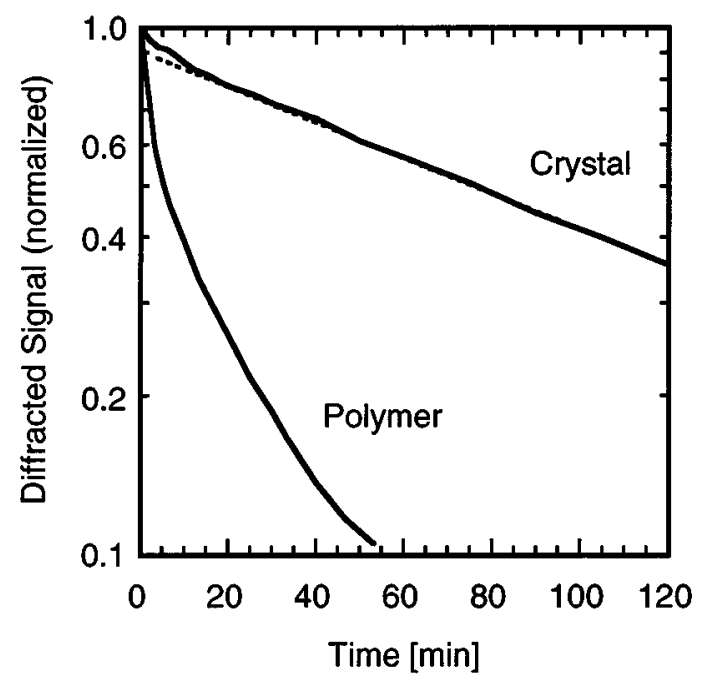

Fig. 6. Experimental data of dark decay in the BisA-NAS:DEH polymer and $\mathrm{BaTiO}_{3}$ crystal at ambient temperature. The dashed line (top left) is a guide for the eye. The grating spacing was $\Lambda=2.62 \mu \mathrm{m}$ and $\Lambda=3.64 \mu \mathrm{m}$, respectively, a dc voltage of $5.0 \mathrm{kV}$ was applied across the polymer, and the probe-beam intensity was less than $4 \times 10^{-7}$ W/ $/ \mathrm{cm}^{2}$. 


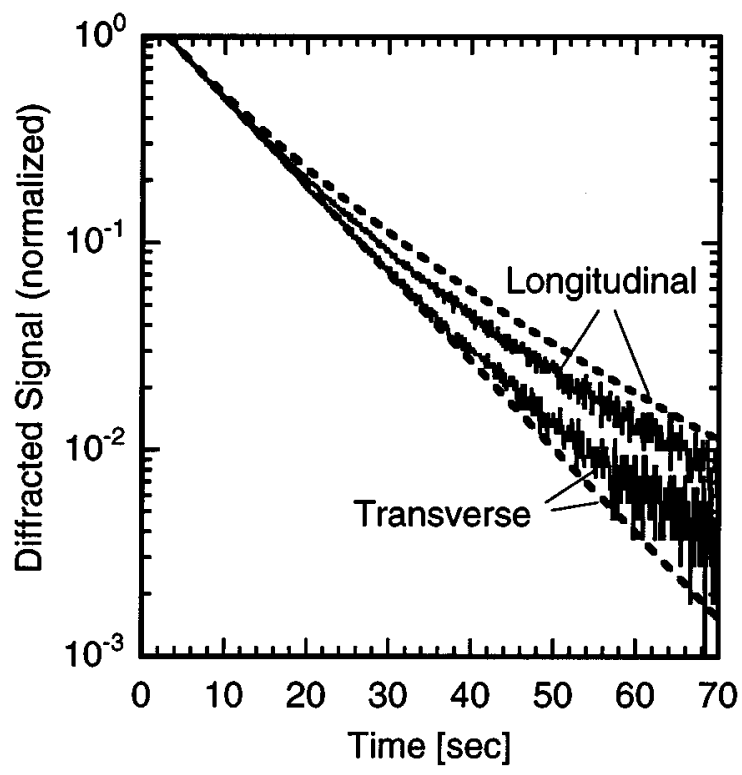

Fig. 7. Experimental erasure data (solid curves) and calculated decay (dashed curves) of the normalized diffracted signal for longitudinal and transverse erasure geometries in $\mathrm{BaTiO}_{3}$ with a $p$-polarized probe beam. The grating spacing was $\Lambda=3.64 \mu \mathrm{m}$, $\alpha L=2.05$ at the erasing beam wavelength $(514.5 \mathrm{~nm})$, no electric field was applied, and the erasing beam intensity was 0.20 $\mathrm{W} / \mathrm{cm}^{2}$.

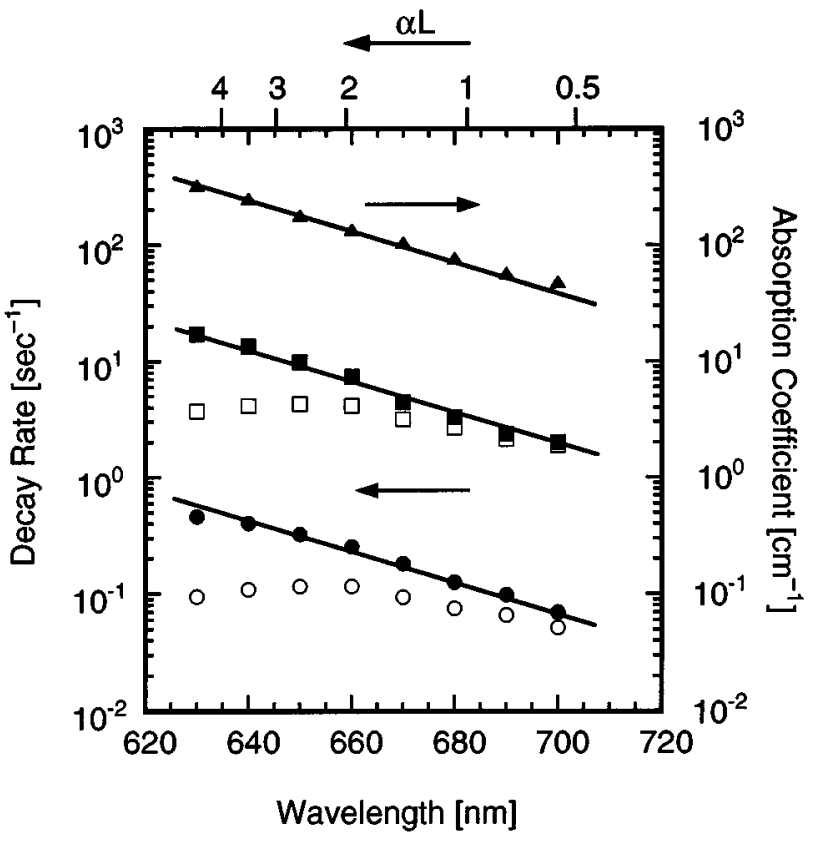

Fig. 8. Attenuation coefficient (triangles), initial apparent decay rates (open circles), and intrinsic decay rates (filled circles) that were corrected for erasing beam attenuation of the polymer BisA-NAS:DEH; decay rates calculated from the photoconductivity data with Eq. (2) (open squares) and corrected for attenuation (filled squares) with Eq. (22). The solid lines are guides for the eye. The probe beam was $p$-polarized, a dc voltage of $5.0 \mathrm{kV}$ was applied, and the peak intensity of the erasing beam with a Gaussian profile at the input face was $0.18 \mathrm{~W} / \mathrm{cm}^{2}$.

cause erasing beam attenuation has a larger effect on the longitudinal erasure. Again, quantitative agreement was not achieved, which implies an intrinsic origin of some of the nonexponential erasure. A plot of the grating erasure in the dark (thermally driven), however, shows that the grating decays exponentially in $\mathrm{BaTiO}_{3}$ (Fig. 6). An explanation for the initial deviation from an exponential decay in the dark was given by Mahgerefteh and Feinberg ${ }^{12}$ and by Tayebati and Mahgerefteh ${ }^{13}$ and is based on the depopulation of shallow traps when the writing beams are turned off and erasure commences in the dark. The erasure experiments were also performed with the erasing beam being present during writing. It was shown ${ }^{12,13}$ that the abrupt change in light intensity when the erasing beam is turned on may cause nonexponential decay. No significant change was noted in our results, however, and we obtained the data reported here by simultaneously turning the writing beams off and the erasing beam on.

The discrepancy between the calculated grating decay incorporating attenuation and Gaussian beam profile and the experimental data with $\mathrm{BaTiO}_{3}$ may be explained if one considers that a uniform erasing beam profile was assumed during numerical computation of the integral in Eq. (18), but the profile was flat only to approximately $10 \%$ across the crystal dimensions. This affects the transverse case much more than the longitudinal case because the probe beam was much smaller than the crystal dimensions. Also, separate measurements of the index of refraction, the angles of incidence, the beam widths, and the attenuation coefficient were necessary to perform the computations. All these quantities contain experimental error, and, e.g., a true attenuation coefficient $10 \%$ lower than the measured value would yield good quantitative agreement in the longitudinal case. Furthermore, reflection of the erasing beam at the back crystal boundary $(\sim 15 \%)$ would result in a faster decay than predicted, inasmuch as reflection was neglected in the calculations, and only partially overlapping writing beams may have resulted in a nonuniform phase grating in the probed volume.

One may obtain a (proper) spectrum of the decay rate with a highly absorbing sample, using Eq. (19) to find the intrinsic speed from a series of grating erasure measurements over a range of wavelengths. Measurements of grating erasure in the photorefractive polymer BisANAS:DEH are shown in Fig. 8, where the measured apparent speed and the calculated intrinsic speed are plotted together with the attenuation coefficient and the speed calculated from the photoconductivity [Eq. (2)], which has also been corrected for attenuation by the approach outlined above [Eq. (22)]. The physical origin of the maximum of the apparent speed and the photoconductivity near $650 \mathrm{~nm}$ is due to the attenuation of the erasing beam because the calculated intrinsic quantities do not exhibit this feature. Figure 8 demonstrates that the intrinsic speed and the photoconductivity are proportional to the attenuation coefficient, which further establishes that the NAS chromophore is responsible for charge generation in the photorefractive polymer, as was previously claimed. ${ }^{11,16,18}$

\section{CONCLUSIONS}

In conclusion, we have found that it is necessary to account for the attenuation and nonuniform intensity pro- 
file of all the beams, particularly of the erasing beam, to understand the time dependence of photorefractive grating erasure even with low attenuation. Nonexponential erasure due to attenuation of the erasing beam should be accounted for before consideration of intrinsic nonexponential decay of the charge grating. We achieved qualitative agreement between theory and experiment when making the simple assumption of intrinsic exponential decay at a rate proportional to the intensity. The results imply that the grating decay in the photorefractive polymer BisA-NAS:DEH is intrinsically nonexponential. This conclusion is supported by the observed nonexponential dark decay and by the observed sublinear intensity dependence of the erasure rate. The calculations presented here were used to explain peculiar features in a spectrum of the decay rates of this photorefractive polymer and to establish that the NAS chromophores are largely responsible for charge generation. Future research should focus on determining the physical origin of the intrinsically nonexponential decay of photorefractive gratings in this polymer. It should be possible to extract the complete intrinsic decay function through deconvolution of the extrinsic decay data.

\section{APPENDIX A: LIST OF ASSUMPTIONS}

The assumptions made in the preceding analysis are explained in detail here. Our analysis was done within the framework of plane-wave first-order coupled-wave theory, and therefore the limitations of this theory apply. ${ }^{5,19}$ The following assumptions and approximations are made within this theory:

(1) The spatial modulation of the index of refraction is sinusoidal along the grating wave vector, and the grating wave vector is confined to the $x-z$ plane.

(2) The analysis is done for thick phase gratings, and only the first diffracted order is considered (Bragg diffraction).

(3) The second derivative of the amplitude is neglected in the wave equations; the amplitude changes little within one wavelength (the slowly varying amplitude approximation).

In addition, the following assumptions and approximations are made:

(4) The reading beam is incident upon the grating at the Bragg angle after one accounts for refraction at the sample boundary.

(5) The reading beam is too weak to cause grating erasure when a separate erasing beam is present. When the reading beam is the erasing beam, Eq. (19) describes the erasure when one chooses the upper sign and $\sigma_{e}=\sigma_{r}$.

(6) Sample birefringence is neglected.

(7) Solutions are obtained in the limit of small diffraction efficiency, which may be achieved by use of a small modulation depth in the interference pattern during writing. This in turn is favorable when one is investigating the dynamics of charge transport, because at large modulation depths the charge distribution becomes nonsinusoidal. ${ }^{3,20}$ A small diffraction efficiency also reduces the effect of coupling between the writing beams; beam coupling during writing introduces distortions in the grating. ${ }^{21}$

(8) Reflection of the erasing beam at the back sample boundary was neglected. Typically, a small percentage of the erasing beam is reflected, which causes the erasure near this boundary to be slightly faster than is assumed in the calculations.

(9) The index grating is uniform over the probed volume. Care was taken in our experiments to probe only the volume of the sample where the grating can be regarded as uniform. The strength of a photorefractive grating depends on the modulation depth of the interference pattern produced by the writing beams, but not on the total light intensity (at least not for samples with a single photorefractive charge trap). Thus attenuation of the writing beams will result in a pronounced nonuniform index grating only when the path lengths of the writing beams differ by more than $0.1 / \alpha$. Because writing beams with Gaussian profiles produce a nonuniform index grating, we used a probe beam having a smaller width than the writing beams when investigating the crystal, whereas the polymer thickness was small enough to allow for good writing beam overlap within the sample in all the cases.

(10) In arriving at Eqs. (19) and (20) it is assumed that the writing beam separation at the sample boundary is small compared with the reading beam width or, more specifically, that $\left[L \sin \left(\theta_{r}-\theta_{s}\right) / c_{s}\right]^{2} \ll \sigma_{r}^{s}$. This approximation results in a convenient analytic solution, but numerical solutions of the more general expressions were within $0.1 \%$ of the approximate solutions of Eqs. (19) and (20).

(11) Wave-front curvature is neglected in the description of the $\mathrm{TEM}_{00}$-mode Gaussian beams. ${ }^{22}$ The Gaussian intensity profile is incorporated, however. Wavefront curvature is small for axial distances $z \ll z_{0}$ $=\pi \sigma_{r}{ }^{2} n / \lambda$. This condition is maintained for all the beams in our measurements because the sample thicknesses $L<5 \mathrm{~mm}$ are much smaller than the focal depth $\left(z_{0}>1.1 \mathrm{~m}\right)$ of the most tightly focused beam. The diffracted signal is affected by wave-front curvature in two ways: through accumulated phase in the coupled-wave equations [Eqs. (10) and (11)] and through Bragg angle mismatch. In our experiments (with $z_{0}>1.1 \mathrm{~m}$ ) the phase error is less than $3 \times 10^{-3} \mathrm{rad}$ at the $1 / e^{2}$ radius, assuming that the beam waist is located in the center of the sample, so that $z<L / 2$. The Bragg angle mismatch is negligible when $\Delta \theta \ll \Lambda_{g} / \pi L$ holds, ${ }^{5}$ where $\Delta \theta$ is the deviation from the Bragg angle because of the angular spread of beams with a Gaussian wave-front curvature. In our experiments $\Delta \theta<4 \times 10^{-6}$ at the $1 / e^{2}$ radius, and $\Lambda_{g} / \pi L>2 \times 10^{-4}$. Similarly, Siegman ${ }^{23}$ showed that a beam with a Gaussian profile may be treated as collimated when $\sigma_{r} / \Lambda_{g} \gg 1$ holds, which is also satisfied in our experiments. Thus we conclude that the phase-front curvature of the $\mathrm{TEM}_{00}$-mode Gaussian beams can be neglected and that the analysis presented in the main body of this paper is applicable under the above experimental conditions. 


\section{ACKNOWLEDGMENTS}

This research was supported by the National Science Foundation Division of Electrical and Communications Systems and by the Nebraska Research Initiative through the Center for Materials Research and Analysis. M. Liphardt was also supported by a University of Nebraska-Lincoln Research and Outreach fellowship funded by the National Science Foundation Division of Education and Human Resources.

Address correspondence to S. Ducharme (e-mail address: ducharme@unlinfo.unl.edu).

\section{REFERENCES}

1. P. Günter and J.-P. Huignard, eds., Photorefractive Materials and Their Applications I, Vol. 61 of Topics in Applied Physics (Springer-Verlag, Berlin, 1988).

2. P. Günter and J.-P. Huignard, eds., Photorefractive Materials and Their Applications II, Vol. 62 of Topics in Applied Physics (Springer-Verlag, Berlin, 1989).

3. N. V. Kukhtarev, "Kinetics of recording and erasing of holograms in electro-optic crystals," Sov. Tech. Phys. Lett. 2, 438 (1976).

4. N. Kukhtarev, V. Markov, and S. Odulov, "Transient energy transfer during hologram formation in $\mathrm{LiNbO}_{3}$ in external applied field," Opt. Commun. 23, 338 (1977).

5. H. Kogelnik, "Coupled-wave theory for thick hologram gratings," Bell Syst. Tech. J. 48, 2909 (1969).

6. M. G. Moharam, T. K. Gaylord, and R. Magnusson, "Bragg diffraction of finite beams by thick gratings," J. Opt. Soc. Am. 70, 300 (1980).

7. J. Baquedano, M. Carrascosa, L. Arizmendi, and J. M. Cabrera, "Erasure kinetics and spectral dependence of the photorefractive effect in $\mathrm{Fe}: \mathrm{LiNbO}_{3}$," J. Opt. Soc. Am. B 4, 309 (1987).

8. R. Jaura, T. J. Hall, and P. D. Foote, "Simplified band transport model of the photorefractive effect," Opt. Eng. 25, 1068 (1986)

9. N. V. Kukhtarev, V. B. Markov, S. G. Odulov, M. S. Soskin, and V. L. Vinetskii, "Holographic storage in electrooptic crystals. I. Steady state," Ferroelectrics 22, 949 (1979).

10. S. Ducharme and J. Feinberg, "Speed of the photorefractive effect in a $\mathrm{BaTiO}_{3}$ single crystal," J. Appl. Phys. 56, 839 (1984).

11. B. E. Jones, S. Ducharme, A. Goonesekera, M. Liphardt, R. V. Athalye, L. Zhang, and J. M. Takacs, "Photoconductivity and grating response time of a photorefractive polymer," J. Opt. Soc. Am. B 11, 1064 (1994).

12. D. Mahgerefteh and J. Feinberg, "Explanation of the apparent sublinear photoconductivity of photorefractive barium titanate," Phys. Rev. Lett. 64, 2195 (1990).

13. P. Tayebati and D. Mahgerefteh, "Theory of the photorefractive effect for $\mathrm{Bi}_{12} \mathrm{SiO}_{20}$ and $\mathrm{BaTiO}_{3}$ with shallow traps," J. Opt. Soc. Am. B 8, 1053 (1991).

14. L. Solymar and D. J. Cooke, Volume Holography and Volume Gratings (Academic, London, 1981).

15. M. Liphardt, A. Goonesekera, B. E. Jones, S. Ducharme, J. M. Takacs, and L. Zhang, "High performance photorefractive polymers," Science 263, 367 (1994).

16. S. Ducharme, R. W. Twieg, J. C. Scott, and W. E. Moerner, "Observation of the photorefractive effect in a polymer," Phys. Rev. Lett. 66, 1846 (1991).

17. K. Buse, S. Riehemann, S. Loheide, H. Hesse, F. Mersch, and E. Krätzig, "Refractive indices of single domain $\mathrm{BaTiO}_{3}$ for different wavelengths and temperatures," Phys. Status Solidi A 135, K87 (1993).

18. S. Ducharme, B. Jones, M. Liphardt, R. Ervin, J. M. Takacs, L. Zhang, and R. Athalye, "Photorefractive properties of bisphenol-A-4,4'-nitroaminostilbene mixed with diethylaminobenzaldehyde-diphenyl hydrazone," in Proceedings of the Topical Meeting on Photorefractive Materials, Effects, and Devices, B. Y. Zel'dovich and E. Krätzig, eds. (Ukrainian Academy of Sciences, Kiev, 1993), p. 1

19. T. K. Gaylord and M. G. Moharam, "Planar dielectric grating diffraction theories," Appl. Phys. B 28, 1 (1982).

20. P. Günter and F. Micheron, "Photorefractive effects and photocurrents in $\mathrm{KNbO}_{3}$ :Fe," Ferroelectrics 18, 27 (1978).

21. V. L. Vinetskii, N. V. Kukhtarev, S. G. Odulov, and M. S Soskin, "Dynamic self-diffraction of coherent light beams," Sov. Phys. Usp. 22, 742 (1979).

22. A. Yariv, Optical Electronics (Holt, Rinehart \& Winston, New York, 1985).

23. A. E. Siegman, "Bragg diffraction of a Gaussian beam by a crossed-Gaussian volume grating," J. Opt. Soc. Am. 67, 545 (1977). 